\title{
EphB4 Regulates Self-Renewal, Proliferation and Neuronal Differentiation of Human Embryonic Neural Stem Cells in Vitro
}

\author{
Tingting Liu ${ }^{\mathrm{a}}$ Xianwei Zeng ${ }^{\mathrm{b}}$ Fangling Sun ${ }^{\mathrm{a}} \mathrm{Hongli} \mathrm{Hou}^{\mathrm{a}}$ Yunqian Guan ${ }^{\mathrm{C}}$ \\ Deyu Guo ${ }^{a}$ Houxi Ai ${ }^{a}$ Guojun Zhang ${ }^{d}$ Wen Wanga,e \\ aDepartment of Experimental Animal Center, Xuanwu Hospital of Capital Medical University, Beijing, \\ ${ }^{b}$ Department of Neurosurgery, The Affiliated Hospital of Weifang Medical College, Weifang, \\ 'Department of Cell Biology, Xuanwu hospital of Capital Medical University, Beijing, 'Beijing Institute of \\ Functional Neurosurgery, Xuanwu Hospital of Capital Medical University, Beijing, 'Beijing Institute for \\ Brain Disorders, Beijing, China
}

\section{Key Words}

EphB4 signaling $\bullet$ Neural stem cells $\cdot$ Self-renewal $\bullet$ Proliferation $\cdot$ Differentiation $・$ Neuron

\begin{abstract}
Background/Aims: EphB4 belongs to the largest family of Eph receptor tyrosine kinases. It contributes to a variety of pathological progresses of cancer malignancy. However, little is known about its role in neural stem cells (NSCs). This study examined whether EphB4 is required for proliferation and differentiation of human embryonic neural stem cells (hNSCs) in vitro. Methods: We up- and down-regulated EphB4 expression in hNSCs using lentiviral over-expression and shRNA knockdown constructs and then investigated the influence of EphB4 on the properties of hNSCs. Results: Our results show that shRNA-mediated EphB4 reduction profoundly impaired hNSCs self-renewal and proliferation. Furthermore, detection of differentiation revealed that knockdown of EphB4 inhibited hNSCs differentiation towards a neuronal lineage and promoted hNSCs differentiation to glial cells. In contrast, EphB4 overexpression promoted hNSCs self-renewal and proliferation, further induced hNSCs differentiation towards a neuronal lineage and inhibited hNSCs differentiation to glial cells. Moreover, we found that EphB4 regulates cell proliferation mediated by the Abl-CyclinD1 pathway. Conclusion: These studies provide strong evidence that fine tuning of EphB4 expression is crucial for the proliferation and neuronal differentiation of hNSCs, suggesting that EphB4 might be an interesting target for overcoming some of the therapeutic limitations of neuronal loss in brain diseases.




\section{Introduction}

Neural stem cells are multipotent, tissue-resident, lineage-restricted neural progenitor cells that are capable of self-renewal as long as they remain undifferentiated [1]. By definition, NSCs have the potential, upon appropriate differentiation steps, to generate the main phenotypes of the central nervous system, i.e., neurons, astrocytes and oligodendrocytes. Therefore, NSCs may have therapeutic value in the treatment of diverse neuronal disorders. NSCs are present in the developing and adult central nervous system (CNS) of mammals [24], specifically in neurogenic areas of the brain, such as the subventricular zone (SVZ) and the subgranular zone (SGZ) [3-6]. Many brain diseases, including stroke and traumatic brain injury, can activate a variety of defense mechanisms aimed at repairing neuronal damage [3, 4]. Mechanisms which regulate proliferation, differentiation and neuronal-glial cell lineage choice of NSCs are important for neuronal restoration [7-9]. The initial signals trigger the mobilization of SVZ- and/or SGZ-resident endogenous NSCs and their migration to the site of injury in order to assist in neuronal regeneration. However, with severe injury, the endogenous attempts at repairing and restoring neuron activity fail to provide neuroprotection. Despite the numerous studies on stem cell therapy, reports on target genes specifically involved in NSCs behaviors are not enough. Therefore, identification of molecular mechanisms that regulate NSCs proliferation and differentiation is urgently needed.

Eph receptors are the largest subfamily of receptor tyrosine kinases, they bind membrane-bound ephrin ligands expressed by neighboring cells and mediate short-range cell-to-cell communication. In mammals, there are nine EphA receptors (EphA1-8 and EphA10) and five EphB receptors (EphB1-4 and EphB6) [10]. Their ligands, the ephrins, are also subdivided into an A-subclass (ephrinA1-ephrinA5), which are tethered to the exoplasmic leaflet of the cell membrane by a glycosylphosphatidylinositol (GPI) anchor, and the B-subclass (ephrinB1-ephrinB3), which contain transmembrane and cytoplasmic regions $[11,12]$. Ephrins and Eph receptors have been implicated in the control of neural stem cell proliferation, survival and differentiation. EphA4 appears to be expressed in the adult neurogenic niches exclusively by neural stem cells, and function to maintain neural stem cell proliferation [13]. Ephrin-A2 is expressed in neural progenitor cells in the SVZ and mediates a reverse signal depending on EphA7 to suppress the proliferation of neural stem/progenitor cells [14]. Intraventricular infusions of the ectodomain of either EphB2 or ephrin-B2 disrupts migration of neuroblasts and increase cell proliferation [15]. Ephrin-B1 is shown to be critical for maintenance of NSCs [16]. Ephrins B2 and B3 and their receptor EphB1 suppress proliferation and survival of NSCs and migration of neuroblasts in the SVZ, rostral migratory stream (RMS) and SGZ $[17,18]$. Clustered EphB2 induces proliferation of SVZ cells in vitro [19]. EphB3 signal suppresses NSCs proliferation in a p53-dependent manner [20]. However, many studies also link the EphB4 receptor to tumor development [21-27]. Based on previous observations, EphB4 may regulate the development of various tumors. Considering that many tumors are sustained by a minor population of tumor-initiating cells that share many properties with stem cells, cancers may arise by transformation of normal tissue stem cells [28]. These suggest that EphB4 might also play important roles in the proliferation and differentiation of NSCs.

Here, we examined the role of EphB4 in regulating the self-renewal, proliferation, differentiation and apoptosis of hNSCs and explored its downstream mediators. Our results show that EphB4 was a key molecular target that regulated proliferation and neuronal-glial cell lineage choice of hNSCs. Its down-regulation impaired self-renewal, proliferation and differentiation towards a neuronal lineage in hNSCs. In contrast, its up-regulation promoted self-renewal, proliferation and differentiation towards a neuronal lineage in hNSCs. Further, we found that the Abl-CyclinD1/CDK4 pathway was involved in EphB4-mediated regulation of hNSCs proliferation but showed no effect on cell differentiation. Moreover, knockdown and overexpression of EphB4 did not affect hNSCs apoptosis.

\section{KARGER}




\section{Cellular Physiology Cell Physiol Biochem 2017;41:819-834

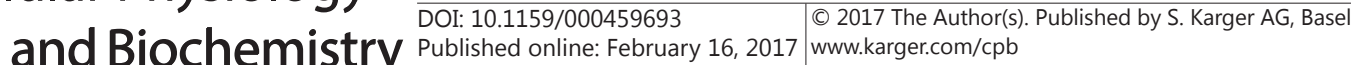

Liu et al.: EphB4 Regulates Proliferation and Differentiation

\section{Materials and Methods}

\section{Isolation and culture of human neural stem cells}

hNSCs isolated from spontaneously aborted fetuses (8-12 weeks) were obtained from the Cell Therapy Center of Xuanwu Hospital of Capital Medical University. The study protocol was approved by the Institutional Review Board (IRB) of Xuanwu Hospital of Capital Medical University. Written informed consent was obtained from patients. The cells that grew as neurospheres were cultured in hNSCs medium consisting of DMEM-F12 (Life Technology, Grand Island, NY, USA), $20 \mathrm{ng} / \mathrm{ml}$ recombinant human basic fibroblast growth factor (bFGF) (Life Technologies), $20 \mathrm{ng} / \mathrm{ml}$ recombinant human epidermal growth factor (EGF) (Life Technologies), 0.5\% N2 (Life Technology), 1\% B27 Supplement (Life Technology), 1\% Glutamax (Life Technology) and 1\% penicillin-streptomycin (Life Technology). After 7-8 days in culture, the neurospheres were dissociated into single cells on poly-ornithine/laminin-coated 6-well plates at a density of $10^{5} / \mathrm{cm}^{2}$ using Accutase (Life Technology) and cultured for 24 hours until the cells had adhered to the plates. Then, we conducted the lentiviral transduction. The cells were grown at $37^{\circ} \mathrm{C}$ in $5 \% \mathrm{CO}_{2}$ and $95 \%$ air with saturated humidity.

\section{Lentiviral transduction and visualization of GFP fluorescence}

Lentiviral-based scrambled-shRNA vectors, EphB4-shRNA vectors, EphB4-overexpression vectors and EphB4-control vectors were all synthesized by Shanghai GeneChem Inc (Shanghai, China). EphB4shRNA driven by the U6 promoter was introduced into the vector GV248, which carries the enhanced green fluorescent protein (EGFP) receptor gene. The target sequence of EphB4-shRNA is GCACCTACGAAGTGTGTGA. The vector GV248, which produces the non-targeting sequence TTCTCCGAACGTGTCACGTT, was used as the negative control (scrambled-shRNA) throughout the study. The overexpression vector GV287 was constructed by driving EphB4 using the ubiquitin promoter, along with EGFP for visualization in cells. A lentiviral vector that expressed EGFP alone was generated as a control vector (EphB4-control). To select effective shRNA sequences targeting EphB4, we transduced NSCs with lentivirus encoding trial shRNA sequences at a multiplicity of infection (MOI) of 4 or with the negative control at a MOI of 2 and then cultured them for 3 days before a 4-day selection period in medium containing $600 \mathrm{ng} \mathrm{ml}^{-1}$ puromycin (Sigma-Aldrich, St. Louis, MO, USA). EphB4 over-expression levels were checked by transducing cells with the GV287 vector at a MOI of 8 or the EGFP control at a MOI of 4. The suppression and upregulation of mRNA expression were analyzed by real-time reverse transcription-polymerase chain reaction (RT-PCR), and EphB4 protein levels were detected using western blotting. The most efficient recombinant vectors were used in subsequent experiments. For co-culture assays, the hNSCs were transduced with lentivirus and cultured for 24 hours at the same MOI as before, and then the medium was replaced with standard medium for another 2 days in culture before the next experiments.

\section{Immunostaining and imaging}

Before immunofluorescence staining, the hNSCs that had been transduced with lentivirus were dissociated into single cells on poly-ornithine/laminin-coated 48 -well plates at a density of 40,000 cells $/ \mathrm{cm}^{2}$ using Accutase (Life Technology) and cultured for 24 hours until the cells fixed on the plates. For 5-bromo2-deoxyuridine (BrdU) detection, cells were cultured for another 48 hours. Then, the culture medium was replaced, $10 \mu \mathrm{M}$ BrdU (Sigma-Aldrich) was added, and cells were incubated for 16 hours before fixation. The numbers of BrdU-positive cells were analyzed using immunocytochemistry. Briefly, hNSCs were fixed with $4 \%$ paraformaldehyde (PFA) for $20 \mathrm{~min}$, then treated with $2 \mathrm{~N} \mathrm{HCl}$ for $17 \mathrm{~min}$ at $37^{\circ} \mathrm{C}$, washed 3 times with phosphate buffered saline (PBS) and blocked for 1 hour at room temperature (RT) with 5\% donkey serum (vol/vol, Jackson ImmunoResearch Laboratories, Philadelphia, PA, USA), Primary mouse anti-BrdU antibody (1:200; Roche, Indianapolis, IN, USA) was added in PBS containing 5\% donkey serum overnight at $4^{\circ} \mathrm{C}$, followed by secondary Cy3-conjugated donkey anti-mouse IgG (1:400, Jackson ImmunoResearch Laboratories) for 2 hours at RT. Then, cells were stained with 4', 6-diamidino-2-phenylindole (DAPI) (1:1000; Sigma-Aldrich) for 30 min at RT and washed with PBS for 3 times. For detection of Ki67, Nestin, cleaved-caspase 3 and caspase 8, cells were cultured for another 48 hours in standard culture medium after adhering to the plates. For detection of differentiation, the medium was changed to culture medium without bFGF and EGF after cells had adhered to the plates. At the $3^{\text {rd }}$ day, we detected doublecortin (Dcx) immunofluorescence. At the $5^{\text {th }}$ day, NG2 immunofluorescence was detected. At the $7^{\text {th }}$ day, the $\beta$ III-tubulin 
and GFAP immunofluorescence were detected. For immunofluorescence staining, cells culture were fixed with 4\% PFA for 20 min, blocked for 1 hour with 5\% donkey serum (vol/vol, Jackson ImmunoResearch Laboratories), permeabilized with $0.1 \%$ Triton X-100 (vol/vol, ZSGB-BiO, Beijing, China), and incubated overnight at $4^{\circ} \mathrm{C}$ with various combinations of the following primary antibodies: rabbit antibody to Ki67 (1:100, Millipore, Temecula, CA, USA), rabbit antibody to Nestin (1:250, Abcam, Cambridge, MA, USA), rabbit antibody to cleaved-caspase 3 (1:400, Cell Signaling Technology, Beverly, MA, USA), rabbit antibody to caspase 8 (1:200, Abcam), rabbit antibody to Dcx (1:100, Santa Cruz Biotechnology, Santa Cruz, CA, USA), goat antibody to NG2 (1:50, Santa Cruz Biotechnology), rabbit antibody to GFAP(1:50, ZSGB-BIO, rabbit antibody to $\beta$ III-tubulin (1:200, Cell Signaling Technology). Samples were subsequently incubated for 2 hours with the appropriate Cy3-conjugated secondary antibodies (1:400, Jackson ImmunoResearch Laboratories). Then, cells were stained with DAPI (1:1000, Sigma-Aldrich) for $30 \mathrm{~min}$ at RT and washed 3 times with PBS. Fluorescent images of cells in monolayers were captured using a NIKON 80i fluorescence microscope. In transduced cells, numbers of cells with positive staining were counted and divided by the total numbers of $\mathrm{EGFP}^{+}$cells. In cells that were not transduced with lentiviral vectors, numbers of cells showing positive staining were counted and divided by the total number of DAPI ${ }^{+}$nuclei. The numbers of positively stained cells were counted from more than 8 non-overlapping fields in each well. For each treatment, 2 different wells were analyzed, and the experiments were repeated more than 5 times.

\section{Real-Time PCR}

For detection of EphB4 and Nestin, total RNAs were extracted from hNSCs that had been transduced with lentivirus using Trizol reagent (TaKaRa Bio, Shiga, Japan) according to the user manual. For detection of differentiation marker, the medium was changed to culture medium without bFGF and EGF after cells had adhered to the plates. At the $3^{\text {rd }}$ day, we extracted total RNA of Dcx. At the $5^{\text {th }}$ day, total RNA of NG2 was extracted. At the $7^{\text {th }}$ day, total RNA of $\beta$ III-tubulin and GFAP were extracted. Then the total RNA were quantified using a NanoDrop 2000 (Thermo Fisher Scientific, Wilmington, DE, USA). Reverse transcription of purified RNA was performed using the PrimeScript RT Master Mix reverse transcription reagent kit (TaKaRa Bio). The quantification of all gene transcripts was performed with a LightCycler 480 Real-Time PCR System (Roche, Indianapolis) using the SYBR Green II real-time PCR kit (TaKaRa Bio). The expression values were normalized against $\beta$-actin. All amplifications were conducted in duplicate, and at least 3 technical and 3 biological replicates were performed.

Real-time PCR primer sequences are as follows: EphB4 F: 5'-CCC ATT TGA GCC TGT CAA TGT C-3', R: 5'-TCA AGC TGC TGG GTG AGG A-3'; Nestin F: 5'-CTC CAA GAA TGG AGG CTG TAG GAA-3', R: 5'-CCT ATG AGA TGG AGC AGG CAA GA-3'; Dcx F: 5'-CGC AAT TCA ATG AGG GCT TTC-3', R: 5'-TTC AGC ACT CAC AGT TCA CAG CA-3'; $\beta$ III-tubulin F: 5'-AAG TAC GTG CCT CGA GCC ATT C-3', R: 5'-GAC CCT TGG CCC AGT TGT TG-3'; NG2 F: 5'-TGC TTT GGA GGC TCT GGA CA-3', R: 5'-GTA TGC AAG CCG ACG CAG AC-3'; GFAP F: 5'-CCA CTT GCA GGA GTA CCA GGA-3', R: 5'-GAA GGT CTG CAC GGG AAT G-3'; $\beta$-actin F: 5'-TGG CAC CCA GCA CAA TGA A-3', R: 5'-CTAAGTCATAGTCC GCCTAGAAGCA-3'.

\section{Western Blots}

Proteins were extracted using radio-immunoprecipitation assay (RIPA) lysis buffer (Applygen, Beijing, China) containing protease inhibitors (Complete mini, Roche). Protein concentrations were calculated using the BCA assay (Applygen). Proteins were separated on SDS polyacrylamide gels and transferred to nitrocellulose membranes (Millipore). Membranes were blocked with 5\% non-fat dry milk for 2 hours at RT and incubated overnight at $4^{\circ} \mathrm{C}$ in $5 \%$ milk/Tris-buffered saline containing $0.1 \%$ Tween-20 (TBST), with the following primary antibodies: mouse monoclonal EphB4 antibody (1:500, Abcam); mouse monoclonal $\beta$ III-tubulin antibody (1:1000, Abcam); rabbit polyclonal Mash1 antibody (1:1000, Abcam); rabbit polyclonal caspase 8 antibody (1:2000, Abcam); rabbit monoclonal CyclinD1 antibody (1:1000, Abcam); mouse monoclonal CDK4 antibody (1:500, Sigma-Aldrich); rabbit polyclonal phospho-Abl (P-Abl) antibody (1:500, Sigma-Aldrich); mouse monoclonal Abl antibody (1:1000, Abcam); rabbit polyclonal phosphoretinoblastoma protein $(\mathrm{pRb})$ antibody $(1: 1000, \mathrm{Abcam})$; mouse monoclonal Tubulin antibody (1:2000, Abcam). The membranes were incubated with appropriate peroxidase-conjugated secondary antibodies 


\section{Cellular Physiology Cell Physiol Biochem 2017;41:819-834

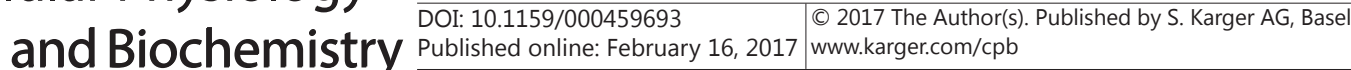

Liu et al.: EphB4 Regulates Proliferation and Differentiation

at 1:2000 for 2 hours at RT, followed by visualization of immunoreactive proteins on film using an ECL kit (Millipore). For each treatment, 6 times were repeated, and experiments were repeated more than 3 times.

\section{Analysis of cloning efficiency}

To assess the frequency of neurosphere formation, neurospheres were mechanically dissociated, carefully counted and plated at 3000 cells per well with $200 \mu \mathrm{l}$ of culture medium in 96-well plates. After 7 days, the number of neurospheres was counted, and the diameters of neurospheres were measured. For each treatment, 6 different wells were analyzed, and the experiments were repeated more than 3 times.

\section{Flow cytometric analysis of apoptosis}

The effect of EphB4 on the apoptosis of hNSCs was measured with a PE-Annexin V apoptosis detection kit (BD Biosciences, Franklin Lakes, NJ, USA). First, hNSCs infected with lentivirus were washed twice with $1 \times \operatorname{PBS}\left(4^{\circ} \mathrm{C}\right)$, followed by resuspension of the cells with $100 \mu \mathrm{l}$ of $1 \times$ Binding Buffer. Next, $5 \mu \mathrm{l}$ of PE-Annexin $\mathrm{V}$ and $5 \mu \mathrm{l}$ 7-AAD were added to the cell suspension for $15 \mathrm{~min}$ in the dark at RT. Finally, $400 \mu \mathrm{l}$ of $1 \times$ Binding Buffer was added to the cells, which were sorted using a FACSCalibur Flow Cytometer (BD Biosciences). Data from $\mathrm{EGFP}^{+} \mathrm{hNSCs}$ were collected and analyzed using CellQuest and ModFIT software. For each treatment, 2 different tubes were analyzed, and the experiments were repeated more than 3 times.

\section{Cell cycle assay}

Cells were collected and washed twice with PBS, resuspended in $500 \mu \mathrm{l}$ of PBS, and fixed by adding $6 \mathrm{ml}$ of $70 \%$ ethanol at $4^{\circ} \mathrm{C}$ for 24-48 hours. The fixed cells were rinsed three times with PBS and stained with a propidium iodide solution containing $10 \mu \mathrm{g} / \mathrm{ml}$ propidium iodide (Biolegend, San Diego, CA, USA) and $0.4 \mathrm{mg} / \mathrm{ml}$ RNase A (QIAGEN, Hilden, Germany) in PBS at $37^{\circ} \mathrm{C}$ for $30 \mathrm{~min}$ in the dark. Samples were evaluated using a FACSCalibur Flow Cytometer (BD Biosciences). Data from EGFP ${ }^{+}$hNSCs were collected and analyzed using CellQuest and ModFIT software. For each treatment, 3 different tubes were analyzed, and the experiments were repeated more than 3 times.

\section{Inhibitor and activator treatments}

To inhibit the protein activity of CyclinD1/CDK4 and Abl before EphB4 overexpression, we added the inhibitors and lentivirus at the same time. After 24 hours, the medium was changed to culture medium with inhibitors only. For analysis of cloning efficiency, cells were then cultured for 6 days before being imaged. Cells that were not transduced with lentiviral vectors were cultured in culture medium with inhibitors for 7 days before being imaged. For BrdU and Ki67 staining, cells were cultured for another 24 hours before being fixed with 4\% PFA. Cells that were not transduced with lentiviral vectors were cultured in culture medium with inhibitors for 48 hours before being fixed with 4\% PFA. For $\beta$ III-tubulin and GFAP staining, cells were cultured for 6 days after viral transduction before being fixed with 4\% PFA. Stock solutions of $10 \mathrm{mM}$ Gleevec (Santa Cruz Biotechnology), an inhibitor of Abl, were prepared by dissolution in dimethyl sulfoxide (DMSO) and were stored at $-20^{\circ} \mathrm{C}$ until use. Then, Gleevec was further diluted in culture medium to treat cells at a final concentration of $1 \mu \mathrm{M}$. DMSO (0.01\%) diluted in the medium was used as the control. Fascaplysin (FAS) (Santa Cruz Biotechnology), an inhibitor of CyclinD1/CDK4, was prepared in DMSO at a stock concentration of $30 \mathrm{mM}$ and stored at $-20^{\circ} \mathrm{C}$, and it was further diluted in cell culture medium to a final concentration of $0.15 \mu \mathrm{M}$. DMSO $(0.0005 \%)$ dilution in the medium was used as the control. To activate the protein Abl, DPH (5-[3-(4-fluorophenyl)-1-phenyl-1H-pyrazol-4-yl]-2,4-imidazolidinedione, Sigma-Aldrich) was prepared in DMSO at a stock concentration of $30 \mathrm{mM}$ and stored at $4^{\circ} \mathrm{C}$, and it was further diluted in cell culture medium to a final concentration of $10 \mu \mathrm{M}$. DMSO $(0.03 \%)$ dilution in the concentration was used as the control. After 2 hours in culture with DPH, cells were fixed using 4\% PFA for immunostaining.

\section{Statistical analysis}

The data are presented as the mean \pm SEM of independent experiments. Statistical analyses were performed using Statistical Package for the Social Sciences (SPSS 13.0 Statistical Software: SPSS Inc., Chicago, IL, USA). Statistical testing was performed using Student's t test to detect differences between groups. Differences were considered statistically significant at ${ }^{*} P<0.05$; ${ }^{* *} P<0.01$; ${ }^{* *} P<0.001$. 


\section{Results}

Establishment of lentivirus-infected cell system

To knock down or overexpress EphB4, lentiviral shRNA vectors targeting the EphB4 gene and an overexpression vector with a cloned EphB4 gene were used in this study. hNSCs were transduced with EphB4-shRNA lentivirus and EphB4-OE lentivirus. Cytoplasmic EGFP was used to identify transduced cells (Fig. 1A). The EphB4 mRNA and protein levels were determined using real-time PCR and western blot. EphB4 mRNA levels and protein expression levels were downregulated by $59.8 \%$ and $64.6 \%$ and upregulated by 115.6 -fold and 2.2-fold, respectively (Fig. 1B, 1C and 1D). We therefore conclude that the lentivirusinfected cell system in hNSCs is effective.

\section{EphB4 regulates the self-renewal and proliferation of hNSCS}

To determine whether EphB4 regulates the self-renewal and proliferation of hNSCs, clone analysis and immunofluorescent labeling were performed on EphB4-deficient and EphB4-overexpressing cells. Compared with the scrambled-shRNA group, EphB4-deficient cells showed a distinct reduction in the neurosphere frequency and clone diameter (Fig. $2 \mathrm{~A}$, upper panel). BrdU staining revealed a decreased number of BrdU $\mathrm{U}^{+}$cells in hNSCs after EphB4 knockdown (Fig. 2B, upper panel). Consistent with this, EphB4 deficiency also reduced the number of Ki67 ${ }^{+}$cells (Fig. 2C, upper panel). Next, we analyzed whether upregulation of EphB4 expression would elicit the opposite effect. hNSCs in the EphB4OE (EphB4-overexpression) group formed more and larger neurospheres than control hNSCs (Fig. 2A, lower panel). Immunostaining for BrdU and Ki67 and quantification of the immunopositive cells confirmed that EphB4 overexpression significantly increased the number of proliferating cells compared to the control group (Fig. 2B, 2C, lower panel).

Fig. 1. Effects of lentivirus transduction on knockdown and overexpression of EphB4 in $\mathrm{hN}$ SCs. (A) Fluorescence images of hNSCs after transduction with scrambled-shRNA, EphB4-shRNA, EphB4-control and EphB4OE lentivirus, as indicated. Note that EGFP fluorescence could be observed in most of the hNSCs. (B) Western blots for EphB4 and tubulin from hNSCs after transduction with the above four lentiviral vectors. (C) Quantification of EphB4 after normalization to tubulin ( $\mathrm{n}=3$ ). (D) Quantitative real-time PCR analysis of the EphB4 mRNA levels after transduction of the four lentivirus vectors $(\mathrm{n}=4) .{ }^{* * *} P<0.001$ with Student's t test; error bars represent the SEM; scale bar $=100 \mu \mathrm{m}$. Abbreviations: Con, control; EGFP, enhanced green fluorescence protein; OE, overexpression; Scr, scrambled; sh, short hairpin.

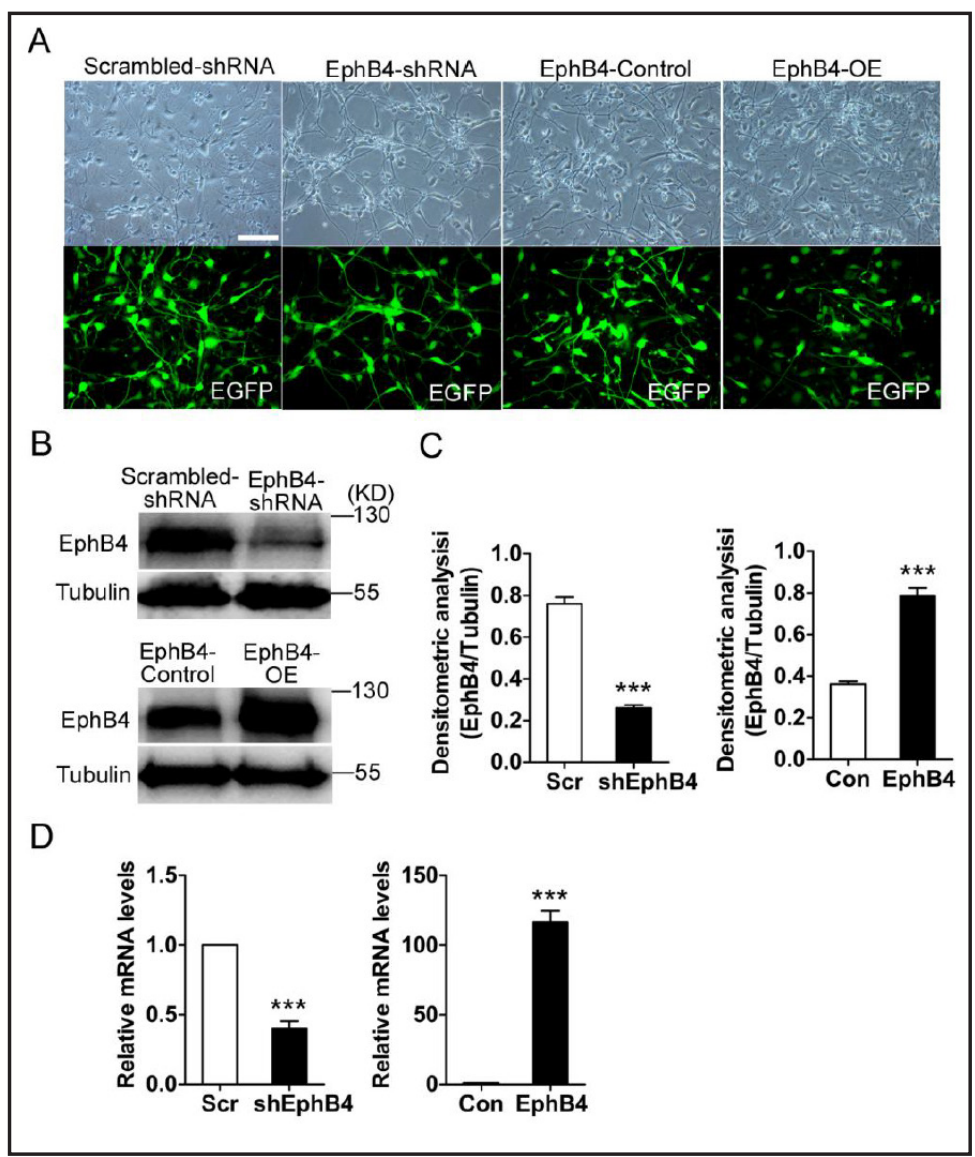


A

Scrambled.enRNA EphB4.shRNA



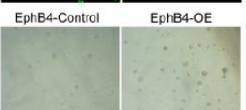
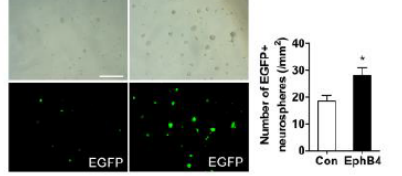

C
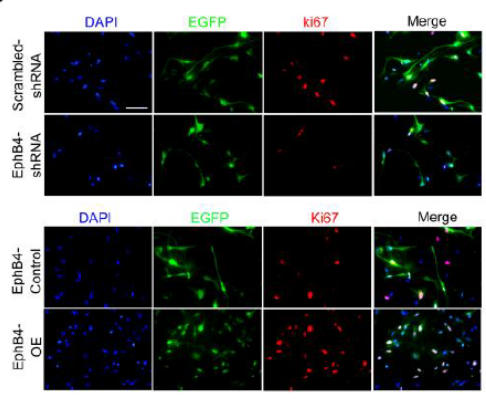
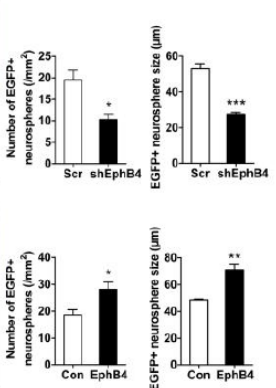

B
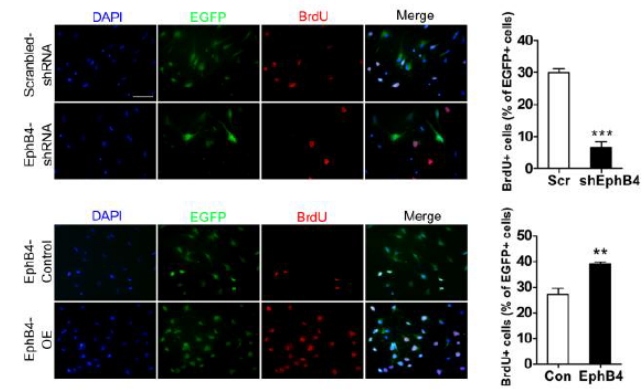

D
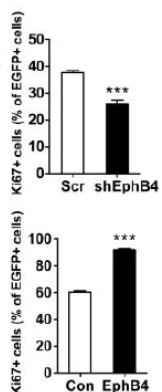

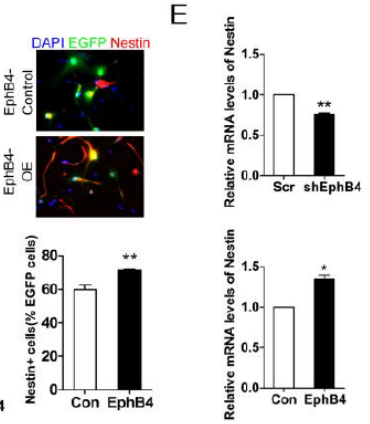

Fig. 2. Effects of EphB4 knockdown and overexpression on the self-renewal and proliferation of hNSCs. (A) hNSCs self-renewal, as assessed by counting the number and measuring the diameter of neurospheres ( $\mathrm{n}=$ 3). (B, C) hNSCs proliferation, as assessed by immunostaining for BrdU and Ki67 in hNSCs ( $=5$ ). (D) Stem cell identity of hNSCs was confirmed with Nestin, a marker of neural stem cells $(\mathrm{n}=5)$. (E) Quantitative real-time PCR analysis of the Nestin mRNA levels after transduction of the four lentivirus vectors $(\mathrm{n}=3)$. ${ }^{*} P$ $<0.05$; ${ }^{* *} P<0.01$; $^{* * *} P<0.001$ with Student's t test; error bars represent the SEM; scale bars $=100 \mu \mathrm{m}$ in A and $50 \mu \mathrm{m}$ in B, C, D. Abbreviations: BrdU, bromodeoxyuridine; Con, control; DAPI, 4',6-diamidino-2-phenylindole; EGFP, enhanced green fluorescence protein; OE, overexpression; Scr, scrambled; sh, short hairpin.

Evidence from in vivo and vitro experiments suggests that Nestin plays an important role in promoting the self-renewal of NSCs $[29,30]$. To further characterize the effects of EphB4 on the self-renewal of hNSCs, we stained hNSCs for Nestin and detected the Nestin mRNA levels. Results show that compared with the scrambled-shRNA group, the number of Nestin $^{+}$cells and Nestin mRNA level were reduced significantly after infection with EphB4shRNA lentivirus. In contrast, Nestin expression and mRNA levels were strongly enhanced by EphB4 overexpression (Fig. 2D, 2E). Thus, in vitro assays revealed that shRNA-mediated EphB4 reduction profoundly impaired hNSCs self-renewal and proliferation and that enhancement of EphB4 expression produced the opposite effects.

\section{Effects of EphB4 on hNSCs differentiation}

NSCs, as self-renewing multipotent cells, have the potential to give rise to differentiated progeny, which is essential for nervous system development. To define the role of EphB4 in hNSCs differentiation, we first performed immunostaining for Dcx and detected its mRNA level using RT-PCR analysis, a neuroblast marker [31], 3 days after incubation in differentiation media. Our results convincingly showed that the number of $\mathrm{Dcx}^{+}$cells and Dcx mRNA level decreased after infection with EphB4-shRNA lentivirus. Conversely, overexpression of EphB4 increased the number of Dcx ${ }^{+}$cells and Dcx mRNA level compared to the control group (Fig. 3A, 3B). Next, we labeled hNSCs with $\beta$ III-tubulin and detected the mRNA level. Similar to the Dcx staining results, EphB4 knockdown and EphB4 overexpression led to decreases and increases, respectively, in the numbers of $\beta$ III-tubulin ${ }^{+}$cells and the mRNA level of $\beta$ III-tubulin. (Fig. 3C, 3D). This indicated that down-regulation of EphB4 impaired neuronal 


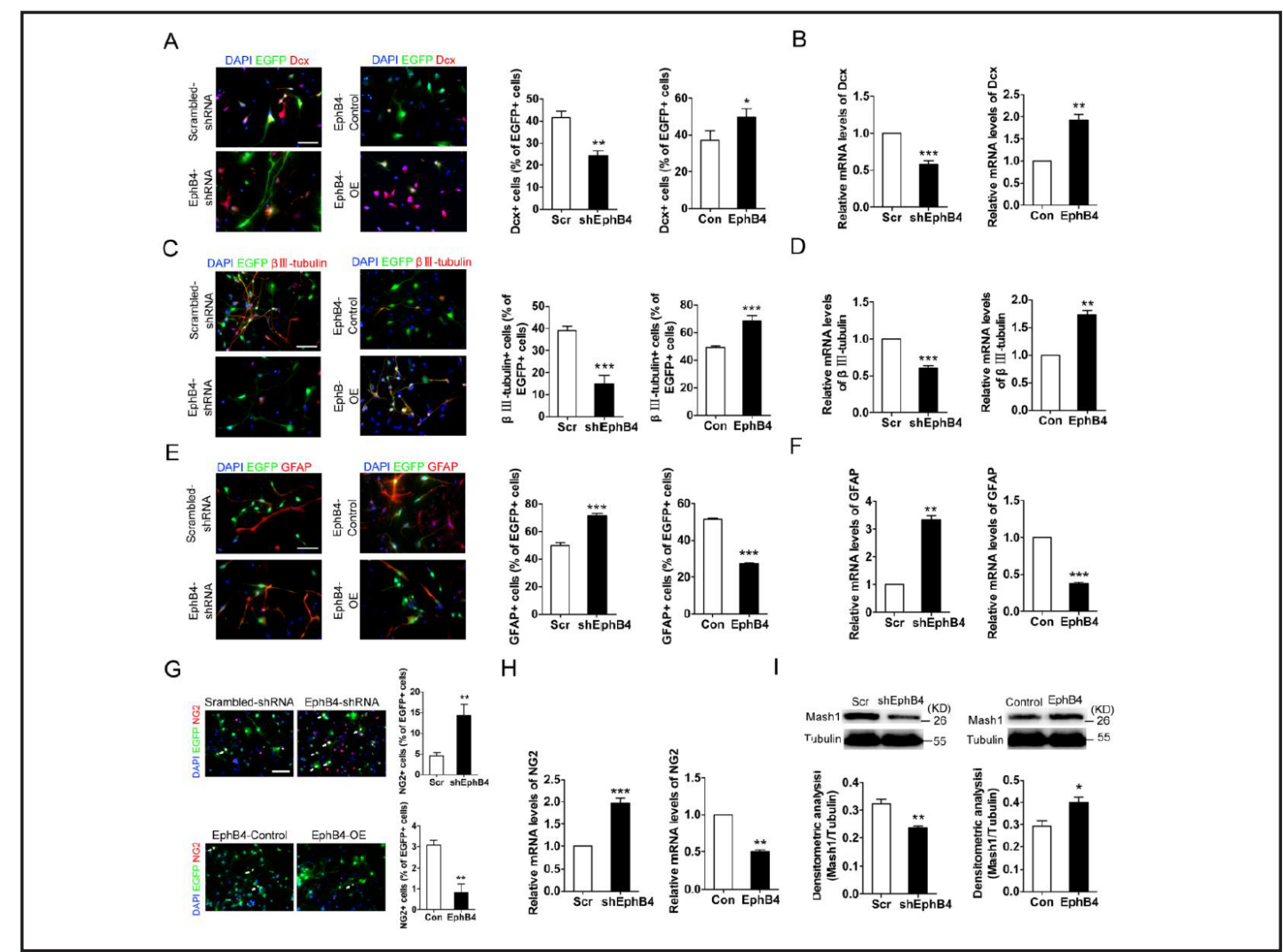

Fig. 3. Effects of EphB4 knockdown and overexpression on differentiation of hNSCs. (A, C, E, G) hNSCs were infected with lentiviral vectors, and immunohistochemical analysis was used to detect the expression of Dcx (A), BIII-tubulin (C), GFAP (E) and NG2 (G) (n = 5). (B, D, F, H) Quantitative real-time PCR analysis of the mRNA levels of Dcx (B), BIII-tubulin (D), GFAP (F), NG2 (H) after transduction of the four lentivirus vectors $(n=3)$. (I) Western blot analysis for Mash1 $(n=3)$. Nuclei were counter-stained with DAPI. The white arrows indicate cells showing positive signals. ${ }^{*} P<0.05$; ${ }^{* *} P<0.01$; ${ }^{* *} P<0.001$ with Student's t test; error bars represent the SEM; scale bar $=50 \mu \mathrm{m}$. Abbreviations: Con, control; DAPI, 4',6-diamidino2-phenylindole; Dcx, doublecortin; EGFP, enhanced green fluorescence protein; GFAP, glial fibrillary acidic protein; OE, overexpression; Scr, scrambled; sh, short hairpin.

differentiation. To further confirm our supposition, we performed western blot analysis of Mash1, an early marker of neuronal differentiation [32] on hNSCs. These results showed that protein expression levels of Mash1 were decreased by knockdown and increased by overexpression of EphB4 (Fig. 3I). Next, we analyzed the impact of EphB4 on glial differentiation. Immunostaining analysis and RT-PCR analysis of glial differentiation markers, GFAP for astrocytes and NG2 for early oligodendrocytes [33] revealed a significantly higher percentage of glial cells derived from hNSCs and a significantly higher of mRNA levels of GFAP and NG2 after EphB4 knockdown than from control hNSCs. Conversely, overexpression of EphB4 decreased the number of $\mathrm{GFAP}^{+}$and $\mathrm{NG}^{+}$cells and the mRNA levels of GFAP and NG2 (Fig. 3E, 3F, 3G, 3H). In conclusion, these results suggest EphB4 was a key molecular switch that regulated differentiation towards neurons or glial cells of hNSCs. Maintenance of EphB4 expression at an appropriate level is crucial for neuronal differentiation.

\section{Effects of EphB4 on hNSCs apoptosis}

To determine the effects of EphB4 on apoptosis, cells subjected to knockdown or overexpression of EphB4 were immunostained for cleaved-caspase 3 and for caspase 8. Quantitative analysis showed that the EphB4-shRNA group and the EphB4-OE group did 




Fig. 4. Effects of EphB4 knockdown and overexpression on apoptosis of hNSCs. (A, B) Immunostaining for cleaved-caspase 3 and caspase 8 to detect apoptotic cells $(n=5)$. (C) Western blot for cleaved-caspase $8(\mathrm{n}=3)$. (D) Quantification of cleaved-caspase 8 after normalization to tubulin $(n=3)$. Nuclei were counter-stained with DAPI. ${ }^{*} P<0.05$; ${ }^{* *} P<0.01$; ${ }^{* * *} P<0.001$ with Student's t test; error bars represent the SEM; scale bar $=50 \mu \mathrm{m}$. (E) Flow cytometric analysis of Annexin V-PE/7-AAD double-stained EGFP ${ }^{+}$cells (n = 3). Abbreviations: Con, control; DAPI, 4',6-diamidino-2-phenylindole; EGFP, enhanced green fluorescence protein; OE, overexpression; Scr, scrambled; sh, short hairpin.

not differ significantly from their respective control groups (Fig. 4A, 4B). The results from western blotting for cleaved-caspase 8 and flow cytometric analysis of apoptosis also showed no differences after knockdown or overexpression of EphB4 (Fig. 4C, 4D, 4E). Thus, these data suggest that apoptosis is not affected by down- or up-regulation of EphB4 expression.

The effects of EphB4 signaling on self-renewal and proliferation are mediated by CyclinD1/ $C D K 4$

To determine how EphB4 regulates proliferation, we used flow cytometric analysis to examine the effects of EphB4 on cell cycle distribution. We found that, compared with the 
Fig. 5. CyclinD1/CDK4 is required for EphB4 to regulate the self-renewal and proliferation of hNSCs. (A) Flow cytometric analysis of the cell cycle in hNSCs infected with scrambled-shRNA, EphB4-shRNA, EphB4-control or EphB4-OE lentivirus $(n=3)$. (B) Western blots for CyclinD1 and CDK4 after transduction with lentiviral vectors $(n=3)$. (C) Western blots for pRb, CyclinD1 and CDK4 after treatment with FAS ( $n=3)$. (D, E) Clone analysis by counting the number and measuring the diameter of neurospheres ( $\mathrm{n}=$ 3). (F, G) hNSCs proliferation, as assessed by immunostaining for BrdU and Ki67 in hNSCs ( $\mathrm{n}=5$ ). ** $P<0.01$; *** $P<0.001$ with Student's t test; error bars represent the SEM. Abbreviations: Con, control; DMSO, dimethyl sulfoxide; FAS, Fascaplysin; OE, overexpression; Scr, scrambled; sh, short hairpin.



scrambled-shRNA group, EphB4 knockdown in hNSCs significantly enhanced the proportion of cells in G0/G1, and this was associated with reduced proportions of cells in S phase and G2/M. Conversely, EphB4 overexpression in hNSCs led to a reduction in the proportion of cells in G0/G1, which was associated with an increased proportion of cells in S phase and G2/M (Fig. 5A). These results indicated that the effects of EphB4 on proliferation may be associated with its regulation of the duration of G0/G1.

CyclinD1 is a member of the G1 cyclin family, and it plays a critical role in promoting G1 or the G1-to-S transition by activating CDK4 [34]. To test our hypothesis, we further investigated whether knockdown or overexpression of EphB4 could influence the expression of CyclinD1 and CDK4. These results showed that EphB4 knockdown in hNSCs reduced the expression of CyclinD1 and CDK4. In contrast, overexpression of EphB4 in hNSCs enhanced the expression of the G1/S-phase markers CyclinD1 and CDK4 (Fig. 5B). Then, we used the CyclinD1/CDK4 inhibitor FAS [35] to suppress CyclinD1/CDK4 activity. The activity of CDK4/ cyclinD1 complex could phosphorylate the retinoblastoma protein $\mathrm{pRb}$, in turn resulting in transcription of genes required for G1-S phase cell cycle progression [36]. We found that the level of $\mathrm{pRb}$ was decreased in FAS group compared to the DMSO group, besides the expression of CyclinD1 and CDK4 were also reduced (Fig. 5C). First, we assessed the effects of FAS on hNSCs self-renewal and proliferation. The number of neurospheres, the clone diameter and the number of BrdU- and Ki67-labeled proliferating cells were significantly 
Fig. 6. The EphB4-induced regulation of cell proliferation via CyclinD1/ CDK4 is mediated by Abl. (A) Western blots for P-Abl and Abl after silencing and overexpression of EphB4 by transduction with lentiviral vectors $(n=3)$. (B, C) Western blots for P-Abl, Abl, CyclinD1 and CDK4 after addition of Gleevec or DPH ( $n=3)$. (D) Effect of Gleevec on the diameter of neurospheres $(n=3)$. (E, F) Effect of Gleevec on proliferation as assessed by immunostaining for BrdU and Ki67 in hNSCs $(n=5)$. (G) Effect of DPH on proliferation as quantified by immunostaining for BrdU and Ki67 in hNSCs $(\mathrm{n}=5)$. ${ }^{* *} P<0.01$; ${ }^{* * *} P<$ 0.001 with Student's t test; error bars represent the SEM. Abbreviations: Con, control; DMSO, dimethyl sulfoxide; FAS, Fascaplysin; Scr, scrambled; sh, short hairpin.



reduced compared to the DMSO group (Fig. 5D, 5E, 5F, 5G). Further, to determine whether CyclinD1/CDK4 mediates the signaling of EphB4, we inhibited CyclinD1/CDK4 using FAS and then overexpressed EphB4 by transducing lentivirus vectors. FAS reduced the selfrenewal and proliferation of hNSCs, which are enhanced by overexpression of EphB4. hNSCs overexpressing EphB4 and treated with FAS showed no difference in the number of neurospheres, the clone diameter or the numbers of BrdU- and Ki67-labeled cells compared to FAS-treated control cells (Fig. 5D, 5E, 5F, 5G). These results demonstrated that effects of up-regulated EphB4 on cell self-renewal and proliferation can be abolished by FAS. Thus, the effects of EphB4 on cell self-renewal and proliferation are mediated by CyclinD1/CDK4.

\section{EphB4 regulates CyclinD1/CDK4 levels and proliferation of hNSCs via Abl}

$\mathrm{Abl}$ regulates breast cancer cell proliferation via binding to EphB4 [25]. Moreover, Abl has been implicated in conveying EphA-mediated axon guidance [37]. Additional knockout studies have demonstrated that $\mathrm{Abl}$ is critical for mammalian neural development [38]. We investigated whether Abl is involved in EphB4-mediated regulation of CyclinD1/CDK4 and cell proliferation in hNSCs. We first found that knockdown of EphB4 resulted in significantly reduced $\mathrm{P}-\mathrm{Abl}$ and $\mathrm{Abl}$ levels in hNSCs. In contrast, $\mathrm{P}-\mathrm{Abl}$ and $\mathrm{Abl}$ levels were increased in EphB4-overexpressing cultures (Fig. 6A). Next, we suppressed Abl activity by using the Abl kinase inhibitor Gleevec (imatinib mesylate) [39]. Addition of Gleevec significantly reduced the expression of P-Abl, CyclinD1 and CDK4 compared to hNSCs treated with DMSO, but there was no effect on Abl (Fig. 6B). We next assessed the effects of Gleevec on hNSCs selfrenewal and proliferation. The clone diameter and the number of BrdU- and Ki67-labeled proliferating cells were reduced to a degree similar to that produced by silencing EphB4 or inhibiting CyclinD1/CDK4 (Fig. 6D, 6E, 6F). Thus, Abl regulates CyclinD1 level and proliferation of hNSCs in a manner similar to that of EphB4 signaling.

Next, to determine whether Abl mediates the signaling downstream of EphB4, we used Gleevec to inhibit Abl activity before overexpressing EphB4 using a lentivirus. Gleevec reduced the self-renewal and proliferation of hNSCs. Importantly, after Gleevec treatment, 
Fig. 7. Differentiation is not mediated by Abl or CyclinD1/CDK4. (A) Effect of Gleevec on neuronal differentiation as assessed by immunostaining for BIII-tubulin $(n=5)$. (B) Effect of Gleevec on astrocytic differentiation as assessed by immunostaining for GFAP ( $n=5)$. (C) Effect of FAS on neuronal differentiation as assessed by immunostaining for $\beta$ III-tubulin ( $\mathrm{n}=$ 5). (D) Effect of FAS on astrocytic differentiation as assessed by immunostaining for GFAP $(n=5)$. Nuclei were counter-stained with DAPI. ${ }^{* *} P<0.01$ with Student's t test, error bars, SEM; scale bar $=50 \mu \mathrm{m}$. Abbreviations: Con, control; DAPI, 4',6-diamidino-2-phenylindole; EGFP, enhanced green fluorescence protein; FAS, Fascaplysin; GFAP, glial fibrillary acidic protein.

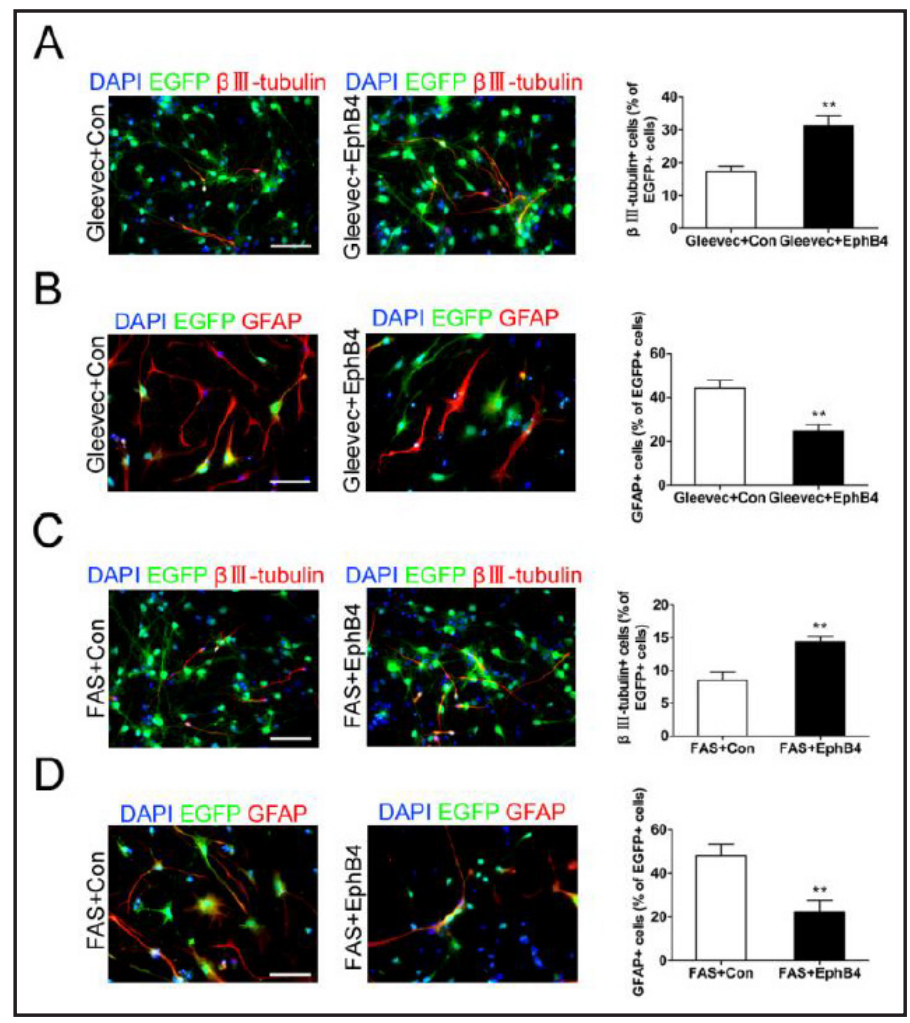

EphB4-overexpressing cells showed no differences from control cells in clone diameter or in the numbers of BrdU-labeled cells and Ki67-labeled cells derived from hNSCs (Fig. 6D, 6E, $6 \mathrm{~F})$. These results demonstrated that the effects of EphB4 overexpression on cell proliferation can be abolished by Gleevec. Further, we used the Abl activator DPH [40] to stimulate Abl activation. Treatment of cells with DPH induced an increase in P-Abl protein expression and there was no effect on Abl. Moreover, DPH also up-regulated the protein expression levels of CyclinD1 and CDK4 (Fig. 6C). Then, using immunostaining for BrdU and Ki67, we observed that DPH promotes cell proliferation (Fig. 6G). Thus, EphB4-mediated signaling to induce cell proliferation goes through Abl to reach CyclinD1/CDK4.

In addition, we investigated whether Abl conveys the EphB4 to regulate the neuronal and glial differentiation of hNSCs. Treatment with Gleevec did not influence the effects of EphB4 overexpression on neuronal differentiation and glial differentiation (Fig. 7A, 7B). Further, we obtained similar results using FAS to inhibit CyclinD1/CDK4 (Fig. 7C, 7D). Thus, EphB4 activates CyclinD1/CDK4 via the Abl pathway to regulate proliferation of hNSCs but does not affect cell differentiation.

\section{Discussion}

We generated a lentivirus-infected cell system that allowed us to up- and down-regulate EphB4 expression in hNSCs. Using this technology allowed us to observe the effects of decreased and increased EphB4 expression in hNSCs and their progeny and in differentiating cells. This makes our system ideally suited to characterizing the influence of EphB4 on the properties of stem cells. We found that EphB4 was a key molecular target that regulated proliferation and neuronal differentiation of hNSCs. Moreover, its effect on the proliferation of hNSCs was mediated by Abl-CyclinD1/CDK4 pathway.

Several EphB tyrosine kinase receptors and their ephrin ligands are involved in regulating the proliferation of neural stem/ progenitor cells $[14,15,17,18]$. However, less is known regarding the effect of EphB4 on NSCs proliferation. In our results, knockdown of 
EphB4 in hNSCs leads to decreased self-renewal and proliferation, as demonstrated by BrdU and Ki67 staining and clone analysis. Previous studies have reported that Nestin is required for the self-renewal of NSCs and that Nestin deficiency leads to impaired self-renewal ability $[29,30]$. Results showed that the number of Nestin-labeled cells and mRNA level of Nestin were also markedly reduced after lentivirus-mediated knockdown of EphB4. Our findings are the first to demonstrate a crucial role for EphB4 in hNSCs proliferation. The role of EphB4 in the regulation of self-renewal and proliferation was further shown by lentivirusmediated EphB4 overexpression, which increased both the numbers and sizes of the clones and increased the numbers of Ki67-, BrdU- and Nestin-positive cells.

To better understand how EphB4 regulates proliferation of hNSCs, we examined the cell cycle distribution of hNSCs after down- and up-regulation of EphB4. We demonstrated that knockdown of EphB4 lengthened the G0/G1 phase of the cell cycle, which was associated with shortening of the S phase and the G2/M phase, whereas overexpression of EphB4 presented the opposite results. Previous studies demonstrated that duration of the G1 phase was linked to cell proliferative behavior [41]. These findings indicated that down- and upregulation of EphB4 might affect cell proliferation by regulating the length of G1. To evaluate this possibility, we assayed the expression of CyclinD1 and CDK4, whose only characterized function is to promote G1 or the G1-to-S transition [34]. Knockdown and overexpression of EphB4 could, respectively, down- and up-regulate the expression levels of CyclinD1 and CDK4. Moreover, inhibition of CyclinD1/CDK4 using FAS reduced the proliferation of hNSCs. Consistent with our results, a previous study showed that down- and up-regulation of CyclinD1/CDK4 could lengthen and shorten G1 to inhibit and promote generation of basal progenitors, respectively [41]. Moreover, inhibiting CyclinD1/CDK4 before upregulating EphB4 blocked the effects of EphB4 overexpression on proliferation. These results indicate that EphB4 may regulate G1/S transitions through CyclinD1/CDK4 to regulate cell selfrenewal and proliferation.

Abl kinases are downstream signalings of EphB4 [25, 42]. Moreover, there is evidence that Abl may have a role to play in stimulation of neurogenesis [43]. We supposed that whether Abl mediated the signaling between EphB4 and CyclinD1/CDK4 to regulate cell proliferation in hNSCs. Results showed that the Abl inhibitor Gleevec reduced the levels of CyclinD1/CDK4 and decreased proliferation in hNSCs, and the effects of Gleevec were equivalent to the effects of silencing EphB4. Moreover, Gleevec also abolished the effects of EphB4 overexpression on proliferation. Further, our results showed that the Abl activator DPH up-regulated the protein levels of CyclinD1 and CDK4 and promoted the proliferation of hNSCs. These results indicate that CyclinD1/CDK4 is the effector of EphB4-mediated cell proliferation and that Abl transduces the EphB4 signaling to CyclinD1/CDK4. Previous studies have shown that EphB2 tyrosine kinase activity regulates cell proliferation through an Abl-CyclinD1 pathway in colon carcinoma [39]. In this study, we first demonstrate that EphB4 regulates cell proliferation in hNSCs through the Abl-CyclinD1/CDK4 pathway and then demonstrate that the effects of EphB4 on proliferation are related to the lengthening of the G1 phase of the cell cycle.

Eph tyrosine kinase receptors and their ephrin ligands have been identified as important regulators of neural stem/progenitor cells differentiation [13, 16, 44-46]. In a prior study, Katakowski et al. reported that EphB2 promotes a neuronal fate in adult subventricular neural precursor cells [19]. However, little is known about a role for EphB4 in hNSCs differentiation. In support of a role of EphB4 in regulating cell differentiation of hNSCs, we show here that knockdown of EphB4 impairs the differentiation of hNSCs toward a neuronal lineage. Moreover, EphB4 silencing promoted the glial differentiation of hNSCs and that overexpression of EphB4 elicited the opposite results. In differentiation cultures, the numbers of Dcx ${ }^{+}$neuroblasts and of $\beta$ III-tubulin ${ }^{+}$neurons, the mRNA levels of Dcx and $\beta$ IIItubulin and the Mash1 levels were reduced. The numbers of $\mathrm{NG}^{+}$cells and $\mathrm{GFAP}^{+}$astrocytes and mRNA levels of NG2 and GFAP were increased in EphB4-silenced hNSCs compared with the scrambled-shRNA group. In contrast, overexpression of EphB4 produced the opposite effects. Previous findings demonstrated that astrocyte-derived ephrinB2 signaling which is 
transduced by EphB4, regulated hippocampal neurogenesis [47]. However, this study did not address the potential for EphB4 to regulate neuronal-glial fate lineage choice of NSCs. We show here for the first time that hNSCs differentiating toward neuronal or glial lineages are highly sensitive to down- and up-regulated expression of EphB4.

We also observed the effects of the Abl kinase inhibitor Gleevec and the CyclinD1/ CDK4 inhibitor FAS on differentiation of hNSCs. Results show that the effects of EphB4 on neuronal and glial differentiation are not mediated by Abl or CyclinD1/CDK4. According to the previous studies on EphB2 [19], it is possible that EphB4 regulate differentiation of hNSCs via Notch1 and Zic1 or other signaling pathways. Identifying downstream signaling pathways that mediate the effect of EphB4 on neuronal-glial fate lineage choice will require further studies. Moreover, although EphB3 and ephrinB3 are implicated in NSCs apoptosis $[18,20,48]$, immunostaining and western blot analysis of cleaved-caspase 3 and caspase 8 as well as flow cytometric analysis of apoptosis demonstrated that down- and up-regulation of EphB4 expression did not affect apoptosis of hNSCs.

In conclusion, we show that down- and up-regulation of EphB4 expression does not affect apoptosis of hNSCs in vitro. Fine tuning of the EphB4 expression level is a viable tool to increase the self-renewal and proliferation capacity of NSCs, and these effects of EphB4 are mediated by the Abl-CyclinD1/CDK4 pathway. Importantly, EphB4 is crucial for the balance between neuronal differentiation and glial differentiation from hNSCs.

\section{Acknowledgments}

We thank Professors Zhiguo Chen and Yumin Luo and their colleagues for excellent technical assistance. This work was supported by the National Science and Technology Major Project (2012ZX09102201-106) and the National Natural Science Foundation of China (81573633, 81503049, 81373994 and 81173575).

\section{Disclosure Statement}

The authors confirm that there are no conflicts of interest.

\section{References}

1 Li M, Liu X, Yue H, Xiong W, Gu J, Xu M: Transplantation of n -acetyl aspartyl-glutamate synthetase-activated neural stem cells after experimental traumatic brain injury significantly improves neurological recovery. Cell Physiol Biochem 2013;32:1776-1789.

$\longrightarrow 2$ Arien-Zakay H, Lecht S, Nagler A, Lazarovici P: Human umbilical cord blood stem cells: Rational for use as a neuroprotectant in ischemic brain disease. Int J Mol Sci 2010;11:3513-3528.

-3 Reekmans K, Praet J, Daans J, Reumers V, Pauwels P, Van der Linden A, Berneman ZN, Ponsaerts P: Current challenges for the advancement of neural stem cell biology and transplantation research. Stem Cell Rev 2012;8:262-278.

4 Yoneyama M, Shiba T, Hasebe S, Ogita K: Adult neurogenesis is regulated by endogenous factors produced during neurodegeneration. J Pharmacol Sci 2011;115:425-432.

$>5$ Madhavan L, Collier TJ: A synergistic approach for neural repair: Cell transplantation and induction of endogenous precursor cell activity. Neuropharmacology 2010;58:835-844.

6 Mathieu P, Battista D, Depino A, Roca V, Graciarena M, Pitossi F: The more you have, the less you get: The functional role of inflammation on neuronal differentiation of endogenous and transplanted neural stem cells in the adult brain. J Neurochem 2010;112:1368-1385.

7 Cheng W, Yu P, Wang L, Shen C, Song X, Chen J, Tang F, Yang Q: Sonic hedgehog signaling mediates resveratrol to increase proliferation of neural stem cells after oxygen-glucose deprivation/reoxygenation injury in vitro. Cell Physiol Biochem 2015;35:2019-2032. 


\section{Cellular Physiology Cell Physiol Biochem 2017;41:819-834 and Biochemistry DOI: 10.1159/000459693 2018 (0)17 The Author(s). Published by S. Karger AG, Basel

8 Huang H, Liu L, Li B, Zhao PP, Xu CM, Zhu YZ, Zhou CH, Wu YQ: Ketamine interferes with the proliferation and differentiation of neural stem cells in the subventricular zone of neonatal rats. Cell Physiol Biochem 2015;35:315-325.

-9 Ke Y, Zhang EE, Hagihara K, Wu D, Pang Y, Klein R, Curran T, Ranscht B, Feng GS: Deletion of shp2 in the brain leads to defective proliferation and differentiation in neural stem cells and early postnatal lethality. Mol Cell Biol 2007;27:6706-6717.

10 Salvucci O, Tosato G: Essential roles of ephb receptors and ephrinb ligands in endothelial cell function and angiogenesis. Adv Cancer Res 2012;114:21-57.

11 Palmer A, Klein R: Multiple roles of ephrins in morphogenesis, neuronal networking, and brain function. Genes Dev 2003;17:1429-1450.

12 Peixoto FO, Pereira-Terra P, Moura RS, Carvalho-Dias E, Correia-Pinto J, Nogueira-Silva C: The role of ephrins-b1 and -b2 during fetal rat lung development. Cell Physiol Biochem 2015;35:104-115.

13 Khodosevich K, Watanabe Y, Monyer H: Epha4 preserves postnatal and adult neural stem cells in an undifferentiated state in vivo. J Cell Sci 2011;124:1268-1279.

14 Holmberg J, Armulik A, Senti KA, Edoff K, Spalding K, Momma S, Cassidy R, Flanagan JG, Frisen J: Ephrin-a2 reverse signaling negatively regulates neural progenitor proliferation and neurogenesis. Genes Dev 2005;19:462-471.

15 Conover JC, Doetsch F, Garcia-Verduqo JM, Gale NW, Yancopoulos GD, Alvarez-Buylla A: Disruption of ephephrin signaling affects migration and proliferation in the adult subventricular zone. Nat Neurosci 2000;3:1091-1097.

16 Qiu R, Wang X, Davy A, Wu C, Murai K, Zhang H, Flanagan JG, Soriano P, Lu Q: Regulation of neural progenitor cell state by ephrin-b. J Cell Biol 2008;181:973-983.

17 Chumley MJ, Catchpole T, Silvany RE, Kernie SG, Henkemeyer M: Ephb receptors regulate stem/progenitor cell proliferation, migration, and polarity during hippocampal neurogenesis. J Neurosci 2007;27:1348113490.

18 Ricard J, Salinas J, Garcia L, Liebl DJ: Ephrinb3 regulates cell proliferation and survival in adult neurogenesis. Mol Cell Neurosci 2006;31:713-722.

19 Katakowski M, Zhang Z, deCarvalho AC, Chopp M: Ephb2 induces proliferation and promotes a neuronal fate in adult subventricular neural precursor cells. Neurosci Lett 2005;385:204-209.

20 Theus MH, Ricard J, Bethea JR, Liebl DJ: Ephb3 limits the expansion of neural progenitor cells in the subventricular zone by regulating p53 during homeostasis and following traumatic brain injury. Stem Cells 2010;28:1231-1242.

21 Alam S, Fujimoto J, Jahan I, Sato E, Tamaya T: Overexpression of ephrinb2 and ephb4 in tumor advancement of uterine endometrial cancers. Ann Oncol 2006;18:485-490.

-22 Ferguson BD, Liu R, Rolle CE, Tan YH, Krasnoperov V, Kanteti R, Tretiakova MS, Cervantes GM, Hasina R, Hseu RD, Iafrate AJ, Karrison T, Ferguson MK, Husain AN, Faoro L, Vokes EE, Gill PS, Salgia R: The ephb4 receptor tyrosine kinase promotes lung cancer growth: A potential novel therapeutic target. PLoS One 2013;8:e67668.

-23 Lee YC, Perren JR, Douglas EL, Raynor MP, Bartley MA, Bardy PG, Stephenson SA: Investigation of the expression of the ephb4 receptor tyrosine kinase in prostate carcinoma. BMC Cancer 2005;5:119.

24 Li M, Zhao ZW, Zhang Y, Xin Y: Over-expression of ephb4 is associated with carcinogenesis of gastric cancer. Dig Dis Sci 2011;56:698-706.

25 Noren NK, Foos G, Hauser CA, Pasquale EB: The ephb4 receptor suppresses breast cancer cell tumorigenicity through an abl-crk pathway. Nat Cell Biol 2006;8:815-825.

-26 Rutkowski R, Mertens-Walker I, Lisle JE, Herington AC, Stephenson SA: Evidence for a dual function of ephb4 as tumor promoter and suppressor regulated by the absence or presence of the ephrin-b2 ligand. Int J Cancer 2012;131:E614-624.

27 Xia G, Kumar SR, Masood R, Koss M, Templeman C, Quinn D, Zhu S, Reddy R, Krasnoperov V, Gill PS: Upregulation of ephb4 in mesothelioma and its biological significance. Clin Cancer Res 2005;11:4305-4315.

28 Kaur S, Roberts DD: Divergent modulation of normal and neoplastic stem cells by thrombospondin- 1 and cd47 signaling. Int J Biochem Cell Biol 2016;81:184-194.

29 Di CG, Xiang AP, Jia L, Liu JF, Lahn BT, Ma BF: Involvement of extracellular factors in maintaining selfrenewal of neural stem cell by nestin. Neuroreport 2014;25:782-787. 


\section{Cellular Physiology Cell Physiol Biochem 2017;41:819-834 and Biochemistry DOI: 10.1159/000459693 2018 (0)17 The Author(s). Published by S. Karger AG, Basel

Liu et al.: EphB4 Regulates Proliferation and Differentiation

30 Park D, Xiang AP, Mao FF, Zhang L, Di C-G, Liu X-M, Shao Y, Ma B-F, Lee J-H, Ha K-S, Walton N, Lahn BT: Nestin is required for the proper self-renewal of neural stem cells. Stem Cells 2010;28:2162-2171.

-31 Francis F, Koulakoff A, Boucher D, Chafey P, Schaar B, Vinet MC, Friocourt G, McDonnell N, Reiner O, Kahn A, McConnell SK, Berwald-Netter Y, Denoulet P, Chelly J: Doublecortin is a developmentally regulated, microtubule-associated protein expressed in migrating and differentiating neurons. Neuron 1999;23:247256.

-32 Torii M, Matsuzaki F, Osumi N, Kaibuchi K, Nakamura S, Casarosa S, Guillemot F, Nakafuku M: Transcription factors mash-1 and prox-1 delineate early steps in differentiation of neural stem cells in the developing central nervous system. Development 1999;126:443-456.

-33 Casamassa A, La Rocca C, Sokolow S, Herchuelz A, Matarese G, Annunziato L, Boscia F: Ncx3 gene ablation impairs oligodendrocyte precursor response and increases susceptibility to experimental autoimmune encephalomyelitis. Glia 2016;64:1124-1137.

-34 Ekholm SV, Reed SI: Regulation of g(1) cyclin-dependent kinases in the mammalian cell cycle. Curr Opin Cell Biol 2000;12:676-684.

-35 Soni R, Muller L, Furet P, Schoepfer J, Stephan C, Zumstein-Mecker S, Fretz H, Chaudhuri B: Inhibition of cyclin-dependent kinase 4 (cdk4) by fascaplysin, a marine natural product. Biochem Biophys Res Commun 2000;275:877-884.

36 Musgrove EA, Caldon CE, Barraclough J, Stone A, Sutherland RL: Cyclin d as a therapeutic target in cancer. Nat Rev Cancer 2011;11:558-572.

-37 Harbott LK, Nobes CD: A key role for abl family kinases in epha receptor-mediated growth cone collapse. Mol Cell Neurosci 2005;30:1-11.

-38 Van Etten RA: Cycling, stressed-out and nervous: Cellular functions of c-abl. Trends Cell Biol 1999;9:179186.

-39 Genander M, Halford MM, Xu NJ, Eriksson M, Yu Z, Qiu Z, Martling A, Greicius G, Thakar S, Catchpole T, Chumley MJ, Zdunek S, Wang C, Holm T, Goff SP, Pettersson S, Pestell RG, Henkemeyer M, Frisen J: Dissociation of ephb2 signaling pathways mediating progenitor cell proliferation and tumor suppression. Cell 2009;139:679-692.

40 Yang J, Campobasso N, Biju MP, Fisher K, Pan X-Q Cottom J, Galbraith S, Ho T, Zhang H, Hong X, Ward P, Hofmann G, Siegfried B, Zappacosta F, Washio Y, Cao P, Qu J, Bertrand S, Wang D-Y, Head MS, Li H, Moores S, Lai Z, Johanson K, Burton G, Erickson-Miller C, Simpson G, Tummino P, Copeland RA, Oliff A: Discovery and characterization of a cell-permeable, small-molecule c-abl kinase activator that binds to the myristoyl binding site. Chem Biol 2011;18:177-186.

41 Lange C, Huttner WB, Calegari F: Cdk4/cyclind1 overexpression in neural stem cells shortens g1, delays neurogenesis, and promotes the generation and expansion of basal progenitors. Cell Stem Cell 2009;5:320331.

42 Nguyen TM, Arthur A, Hayball JD, Gronthos S: Ephb and ephrin-b interactions mediate human mesenchymal stem cell suppression of activated t-cells. Stem cells Dev 2013;22:2751-2764.

43 Schlatterer SD, Suh HS, Conejero-Goldberg C, Chen S, Acker CM, Lee SC, Davies P: Neuronal c-abl activation leads to induction of cell cycle and interferon signaling pathways. J Neuroinflammation 2012;9:208.

-44 Doeppner TR, Bretschneider E, Doehring M, Segura I, Senturk A, Acker-Palmer A, Hasan MR, ElAli A, Hermann DM, Bahr M: Enhancement of endogenous neurogenesis in ephrin-b3 deficient mice after transient focal cerebral ischemia. Acta Neuropathol 2011;122:429-442.

-45 Hara Y, Nomura T, Yoshizaki K, Frisen J, Osumi N: Impaired hippocampal neurogenesis and vascular formation in ephrin-a5-deficient mice. Stem Cells 2010;28:974-983.

46 Shu Y, Xiao B, Wu Q, Liu T, Du Y, Tang H, Chen S, Feng L, Long L, Li Y: The ephrin-a5/epha4 interaction modulates neurogenesis and angiogenesis by the p-akt and p-erk pathways in a mouse model of tle. Mol Neurobiol 2016;53:561-576.

47 Ashton RS, Conway A, Pangarkar C, Bergen J, Lim KI, Shah P, Bissell M, Schaffer DV: Astrocytes regulate adult hippocampal neurogenesis through ephrin-b signaling. Nat Neurosci 2012;15:1399-1406.

48 Theus MH, Ricard J, Glass SJ, Travieso LG, Liebl DJ: Ephrinb3 blocks ephb3 dependence receptor functions to prevent cell death following traumatic brain injury. Cell Death Dis 2014;5:e1207. 